\title{
La red de atención a la salud mental correspondiente al hospital universitario Príncipe de Asturias en Madrid (España)
}

\author{
Alberto Fernández Liria \\ Hospital Universitario Principe de Asturias \\ Universidad de Alcalá de Henares \\ Ana Moreno Pérez. \\ Hospital Universitario Príncipe de Asturias \\ Juan González Cases \\ Centro de Rehabilitación Psicosocial de Alcalá. Consejería de Familia y Asuntos Sociales. Comunidad de Madrid (Gestión técnica \\ Grupo Exter S.A.)
}

\begin{abstract}
Resumen
Se describe la estructura y funcionamiento de una red pública de atención a la salud mental que cubre una población de 450.000 habitantes de la zona metropolitana este de Madrid. Se trata de una red que funciona como parte de un sistema público, universal y gratuito en el momento del pago, aunque tiene varios financiadores y parte de los proveedores son instituciones de titularidad privada que se financian mediante un concierto con la administración pública. Integra 26 dispositivos en los que trabajan 229 profesionales asignados a equipos interprofesionales. Además de las funciones asistenciales, la red desarrolla labores de docencia e investigación.
\end{abstract}

Palabras claves: sistemas de atención a la salud mental; red de salud mental; salud mental comunitaria.

\begin{abstract}
The network of mental health care in the hospital universitário Principe de Asturias in Madrid (Spain). We describe the structure and functioning of a public mental health care network that covers a population of 450.000 inhabitants in the metropolitan east area of Madrid. It is a network that operates as part of a public, universal and without payment at the time of being attended National Health Service, although it is granted from several different public founders and some of the providers are private institutions employed by the public system. It includes 229 mental health professionals work in 26 integrated inter-professional teams. In addition to the functions of care, the network develops teaching and research work.
\end{abstract}

Keywords: mental health care systems; mental health network, community mental health.

$\mathrm{E}$ n España existe un Sistema Nacional de Salud que oferta atención gratuita en el momento del uso a todas las personas que presentan necesidades de atención sanitaria. El Sistema está integrado por los Servicios de Salud de las 17 comunidades autónomas (que funcionan, de hecho como estados federados) cuyos gobiernos se encargan de la gestión de los mismos. El Estado central se reserva sólo funciones de coordinación e inspección (Ley General de Sanidad, 1986). Esta atención incluye la atención a la salud mental incluidas las intervenciones psicosociales como la rehabilitación y la psicoterapia (Ministerio de Sanidad y Política Social, 2009).

El actual modelo de salud mental se remonta a mitad de la década de los 80 cuando se oficializa la reforma psiquiátrica en España. Esta reforma conlleva la transformación de un modelo básicamente marginal, disgregado y manicomial hacia otro modelo de atención a la salud mental de carácter comunitario. La filosofía que subyace a esta nueva propuesta de atención es que las personas con problemas psiquiátricos no deben ser excluidas de su comunidad y por tanto la atención se les debe prestar lo más cerca de su entrono. Dicho abordaje reconoce también que las necesidades de estas personas en su funcionamiento e integración en la comunidad son múltiples y variadas, algunas sanitarias pero otras trascienden el ámbito sanitario y se manifiestan en dimensiones sociales y psicosociales, Como señala Desviat (2001, p. 131): "la Salud Mental Comunitaria va a designar un modelo de atención caracterizado por varios desplazamientos: a) del sujeto enfermo a la colectividad; $b$ ) del espacio hospitalario (sobre todo el manicomial) a la comunidad; del psiquiatra al equipo". En otro documento (Desviat, 2000), el mismo autor señala las características que sirven para analizar cualquier proceso de reforma psiquiátrica, pues la reforma psiquiátrica española reúne, en su inicio, las condiciones que han estado presentes en casi todos los procesos de reforma con la ventaja de haber empezado tarde el proceso y el conocimiento previo de buena parte de las dificultades encontradas en otros países: Un momento histórico muy apropiado (una sociedad receptiva, 
movilizada socialmente); Un proceso de reforma sanitaria acorde con los principios fundamentales de la reforma psiquiátrica (Servicio Nacional de Salud, universalidad, integración de redes, descentralización, potenciación de Atención Primaria, desarrollo de Servicios Sociales de base); Núcleos profesionales preparados para la reforma con implantación profesional y social (había existido un conjunto de experiencias, un movimiento previo); Un amplio consenso programático: expresado en el artículo 20 de la Ley General de Sanidad (1986); Escasa legitimidad por su vinculación franquista de la psiquiatría tradicional (tanto universitaria como asistencial); Una administración dispuesta a asumir la reforma psiquiátrica (el partido en el gobierno desde 1982 necesitaba de una reforma de las prestaciones sanitarias y de servicios sociales para su propia legitimación como partido de cambio social, como partido socialdemócrata).

Considera Desviat (2000) que la reforma nace en un momento de profundas trasformaciones sanitarias y sociales, en tiempos ilusionados de reconstrucción democrática de la sociedad, de la cosa pública, de la trama social. El proceso desinstitucionalizador, alternativo, comunitario, forma parte de una época singular: donde el horizonte era el Estado de bienestar, la universalización de las prestaciones sanitarias y sociales, su equidad: reforzar el lazo social por medio de una redistribución social de la renta.

Las premisas de esta reforma y del modelo de atención en salud mental resultante se pueden resumir en (adaptado de Salvador-Carulla et al., 2002):

a) Integración de la atención a los trastornos mentales por ley en el sistema sanitario general.

b) Integración funcional de todas las redes de servicios psiquiátricos en una sola red.

c) Creación de sistemas de coordinación y enlace con Atención Primaria y Servicios Sociales.

d) Provisión de servicios para áreas geográficas definidas coincidentes con las áreas sanitarias.

e) Creación de equipos multidisciplinarios.

f) Provisión de recursos y dispositivos comunitarios sanitarios y sociales.

g) Puesta en marcha de programas para grupos diagnósticos específicos.

h) Desarrollo de una estructura administrativa para el desarrollo, monitorización y evaluación sistemática de la reforma.

i) Formación de los profesionales.

La organización administrativa descentralizada del Estado ha propiciado diferentes desarrollos del modelo de salud mental comunitario diseñado tras la reforma psiquiátrica en España. Estos diferentes modelos, aun compartiendo y manteniendo los elementos nucleares que sustentaron la reforma, han producido tras más de dos décadas de desarrollo diferentes desajustes explicitados abajo.

El gran crecimiento de recursos sanitarios y sociales para atender las diferentes necesidades de este colectivo, que se ha plasmado en redes complejas de servicios y prestaciones ha causado a veces dificultades de coordinación y sinergia entre los dispositivos.

A pesar del incremento de recursos y prestaciones experimentado en la atención en salud mental, la demanda se ha disparado por encima de la oferta de servicios de tal forma que aún siendo muchos y variados los recursos existentes, resultan insuficientes para atender de forma adecuada a la población.

Viraje de la atención comunitaria hacia modelos más centrados en el hospital.

Auge de la perspectiva biológica en detrimento de perspectivas más globales e integradoras de corte bio/piso/social.

Debilitamiento y retroceso de las políticas generales de salud pública, enfoques comunitarios, sistemas de protección social, etc. que afectan de forma sustancial al modelo de salud mental comunitario.

A pesar de las sombras que amenazan al modelo de salud mental comunitario se presenta a continuación una plasmación concreta de dicho modelo en un territorio de la Comunidad de Madrid.

La red de servicios de salud mental correspondiente al Hospital Universitario Príncipe de Asturias proporciona atención a la salud y los trastornos mentales de un área geográfica de $450.000^{1}$ habitantes divididos en dos grandes municipios industriales de la periferia de Madrid y algunos pequeños municipios aledaños con un perfil de población residencial, rural o semi-rural. La figura 1 representa los elementos de la red de atención a la salud mental. Cada uno de los dispositivos está conformado por un equipo interprofesional. La red integra en la actualidad 229 profesionales de plantilla más 30 residentes (16 de Psiquiatría, 16 de Psicología Clínica y 6 de Enfermería Especialista en Salud Mental).

La puerta de entrada para la inmensa mayoría de pacientes es a través de la atención primaria de salud, aunque para un 5\% de los casos puede ser la urgencia hospitalaria o la derivación desde otras especialidades médicas o - muy excepcionalmente - desde instancias judiciales. Todos los pacientes atendidos tienen un facultativo de referencia en el centro de salud mental correspondiente. Los pacientes que requieren simultánea o sucesivamente del uso de más de uno de los dispositivos de la red tiene asignado un coordinador de cuidados que se encarga de guiarle para lograr el mejor aprovechamiento de la oferta asistencial y de coordinar la actuación de los profesionales encargados de ese paciente en los dispositivos que usa para hacerlos actuar sinérgicamente en un plan individualizado de atención

\section{Dispositivos que integran la red}

Dispositivos sanitarios. En realidad todos los dispositivos del área incluyen elementos de tratamiento, rehabilitación, cuidados y apoyo social y tienen una naturaleza mixta que incluye elementos estrictamente sanitarios, más psicosociales y puramente de apoyo. En el listado que sigue, sin embargo, clasificaremos los dispositivos de acuerdo con su naturaleza predominante según un esquema en el que las columnas de la figura 1 se situarán de izquierda a derecha desde un polo más "sanitario", orientados, sobre todo al tratamiento, pasando por dispositivos más puramente "rehabilitadores" hasta un polo más de "apoyo social". 


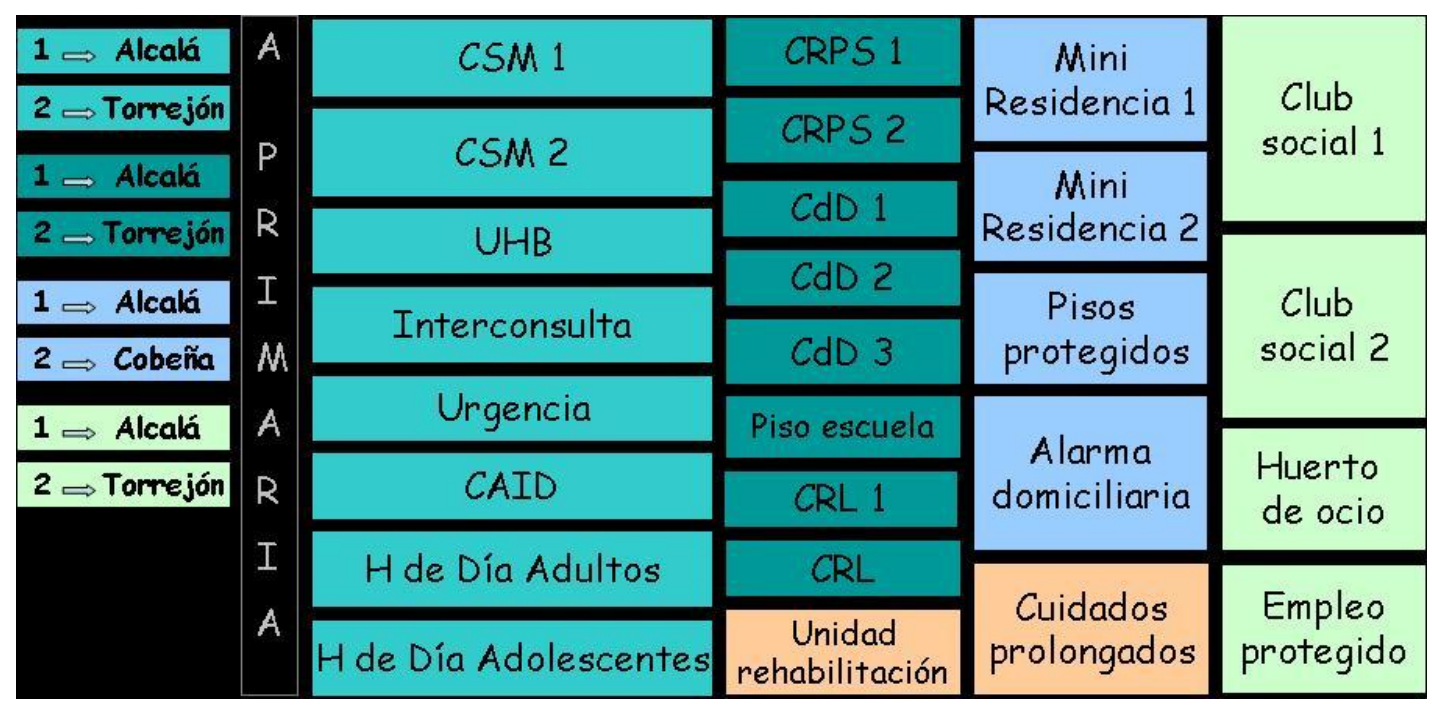

Figura 1. Dispositivos que constituyen la red de atención a la salud mental correspondiente al Hospital Universitario Príncipe de Asturias

El papel de la atención primaria de salud: dos modelos de trabajo. La puerta principal de entrada a la red es la Atención Primaria de Salud. Se calcula que un $30 \%$ de las personas que habitan en el área acuden anualmente al médico de atención primaria presentando un problema de salud mental que requeriría atención (Goldberg \& Huxley, 1980, 1992; Kessler \& Üstün, 2008). La mayor parte de estos problemas son atendidos y resueltos por los profesionales de atención primaria. Cuando la intervención requerida excede la capacidad de estos profesionales, lo derivan al centro de salud mental. Anualmente un $2 \%$ de esta demanda es atendida en los servicios de atención especializada del área población (durante el año 2010, se realizaron 48240 intervenciones ambulatorias y se atendieron un total de 4696 nuevas consultas. Se atendieron 3179 urgencias en el hospital de los que 271 precisaron ingreso, generando un total de 5899 estancias; en programas de día se atendieron a 432 personas).

Tradicionalmente los pacientes han sido derivados desde la atención primaria de salud a través de un sistema informático y mediante un informe en el que se especifica, al menos el motivo de la derivación con el que llegan al Centro de Salud Mental. A partir del año 2006 comenzó a desarrollarse de forma piloto un nuevo sistema por el que los psiquiatras y psicólogos que van a recibir a los pacientes propuestos por los médicos de atención primaria se desplazan para hacerlo al centro de salud en el que trabajan estos, de modo que pueden verlos conjuntamente o, por lo menos, hablar sobre ellos en la reunión conjunta que se celebra al final de la mañana. En los primeros análisis la experiencia ha mejorado la satisfacción de los profesionales de ambos niveles de atención y disminuido el número de pacientes derivados, y la lista de espera y mejorado los criterios de derivación (Hornillos Jerez, Rodríguez Pedraza, González Aguado, \& Fernández Liria, 2009). Desde septiembre de 2008 la experiencia se ha extendido a dos centros más de salud y actualmente se prevé la generalización de este modelo de trabajo que permite acercar a los profesionales de los niveles de atención primaria y especializada.
Los Centros de salud mental. La red integra dos centros de salud mental. Son el elemento central de la red. En ellos se basan los programas de evaluación de problemas de salud mental, atención ambulatoria, atención ambulatoria urgente, atención domiciliaria, coordinación y apoyo con atención primaria, coordinación y apoyo con Servicios Sociales y Rehabilitación. Constituyen la vía principal de entrada a la red, trabajando en segunda línea respecto a la Atención Primaria de Salud.

Todos los pacientes atendidos en la red tienen un facultativo de referencia en el Centro de salud mental. El personal que trabaja en estos es personal público, que depende en su mayor parte de la Gerencia del Hospital Universitario Príncipe de Asturias, aunque hay quien depende de la Gerencia de Atención Primaria. Tienen habilitados turnos de mañana y tarde. Están conformados por equipos multiprofesionales que integran 4.6 psiquiatras, 1.7 psicólogos, 1.3 enfermeros y 0.8 trabajadores sociales por cada 100.000 habitantes más el necesario apoyo administrativo y de otras profesiones.

De ellos un $14 \%$ de los psiquiatras, un $50 \%$ de los psicólogos y un $25 \%$ de los trabadores sociales trabajan con niños y adolescentes (menores de 18 años). El 95\% de los pacientes atendidos es derivado por atención primaria (el resto procede de la urgencia u otros servicios del hospital) directamente a través de un sistema de telecita. Con algunos equipos de atención primaria de Alcalá se está desarrollando un programa de especialista consultor por el que los facultativos de los Servicios de Salud Mental se desplazan al centro de salud a valorar los pacientes nuevos y prestar apoyo a los profesionales de primaria(Hornillos Jerez et al., 2009).

\section{Centro de atención integral a las drogodependencias}

$\mathrm{La}$ atención a las drogodependencias se presta por regla general en la Comunidad de Madrid desde una red que es independiente y funciona en paralelo a la red de atención a la salud mental. El Centro de Atención a las Drogodependencias de Alcalá está integrado en la red de atención a la salud mental 


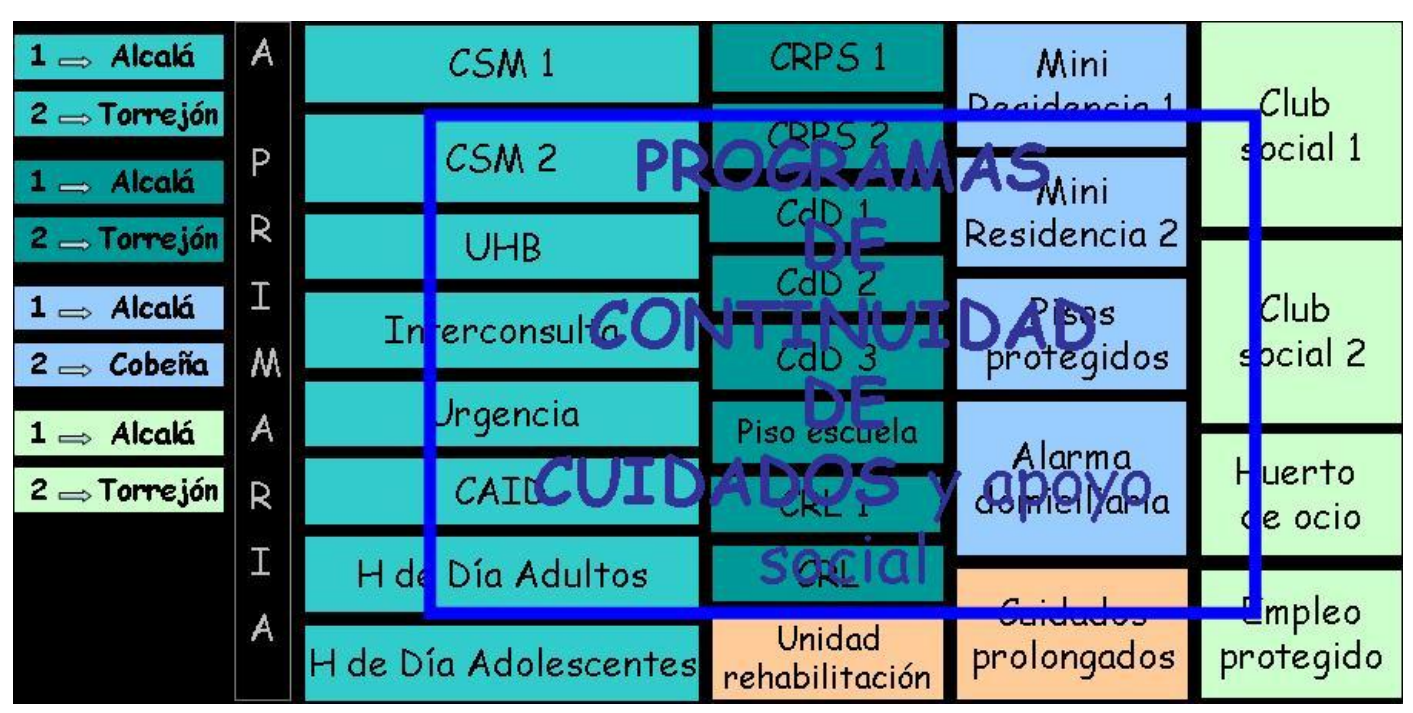

Figura 2. Dispositivos que constituyen la red de atención a la salud mental en correspondiente al Hospital Universitario Príncipe de Asturias con representación del programa de continuidad de cuidados.

desde 1991 por un acuerdo que dio lugar a un programa piloto. Proporciona tratamiento a personas que presentan consumos problemáticos de sustancias o trastornos adictivos, así como, orientación y apoyo a familiares. Además, cuenta con el Servicio de Prevención de drogodependencias dirigido a población general. Está financiado en parte, por la Agencia Antidroga de la Comunidad de Madrid a través de un convenio que mantiene con el Ayuntamiento de Alcalá de Henares. El equipo interprofesional está integrado por 4 psicólogos, 1 psiquiatra, 2 médicos, 1 trabajadores sociales, 2 enfermeros, 1 terapeuta ocupacional y el necesario apoyo administrativo y logístico.

\section{Unidad de hospitalización}

Está situada en la cuarta planta del Hospital Universitario Príncipe de Asturias. Tiene 18 camas, distribuidas en 10 habitaciones individuales y 4 dobles. Tiene dos salas de estar comunes, cuatro despachos clínicos, sala de reuniones y espacio para enfermería. La tasa de camas es de 4 por 100.000 habitantes, la tercera parte de la media de Madrid reflejada en el Plan de Salud Mental de 2003. La plantilla integra 3 psiquiatras, 11 enfermeros, 10 auxiliares de clínica, 6 celadores y un monitor de terapia ocupacional. Esto permite garantizar que en los momentos de menos personal hay, además de un psiquiatra y dos médicos residentes que integran el equipo de guardia, al menos dos diplomados en enfermería y dos auxiliares a los que se suma un celador. La unidad funciona como un medio terapéutico que se articula a través de una serie de reuniones diarias del equipo y de actividades grupales de los pacientes que incluyen diariamente un grupo de buenos días, un grupo de articulación del medio terapéutico y actividades ocupacionales. La estancia media en el año 2010 fue de 20 días. Desde el año 2010 la unidad, así como el Programa de Interconsulta y Enlace y el Hospital de Día, cuenta con un certificado de implantación de un sistema de gestión de la calidad como medio terapéutico a través de la norma ISO 9001:2008.

Programa de interconsulta y enlace para pacientes ingresados o atendidos por otras patologías
Se lleva a cabo por un psiquiatra y varios residentes de Psiquiatría y Psicología Clínica, desde una zona de despachos habilitada en la cuarta planta del Hospital Universitario Príncipe de Asturias, fuera de la Unidad de Hospitalización, junto a la Oficina de Coordinación. Incluye, la atención a los pacientes ingresados por el Programa de Atención a los Trastornos de la Conducta Alimentaría que ingresan a cargo de Psiquiatría en las camas de Endocrinología y Nutrición en la tercera planta del hospital, la Interconsulta a demanda desde los demás servicios del hospital y los programas de enlace desarrollados con otros servicios (Como oncología, reumatología, la unidad del dolor).

\section{Urgencia}

Los servicios proporcionan atención urgente de 8:00h. a 15:00h. en los centros de salud mental y 24 horas al día siete días en semana en el servicio de urgencias del Hospital Universitario Príncipe de Asturias. Este último servicio lo atienden de 8:00h. a 15:00h. un psiquiatra del hospital y de 15:00h. a 8:00h. los psiquiatras del área, de cualquiera de las dos entidades proveedoras, que actúan por turno, como psiquiatras de guardia junto con dos residentes de Psiquiatría. En el servicio de urgencias del hospital se atienden una media de 9 urgencias diarias que generan 0.75 ingresos al día.

\section{Hospital de Día para adultos}

Situado en la primera planta de un antiguo pabellón escolar en cuya planta baja está el hospital de día para adolescentes. Atiende sobre todo pacientes con trastornos graves de la personalidad proporcionándoles un medio terapéutico que se trabaja con una orientación interpersonal con foco especial en la capacidad de mentalización. Proporciona tratamiento psicoterapéutico intensivo de lunes a viernes en horario de 9:00h. a 16:00h. a pacientes que han sido derivados allí desde los Centros de Salud Mental. Tiene 20 plazas. La estancia media es entorno a los 11 meses. El equipo está integrado por dos psiquiatras, un enfermero, un terapeuta ocupacional y un monitor de terapia ocupacional, además del apoyo administrativo y logístico (incluido el servicio de catering que permite a los 
pacientes comer en el centro) que comparten con el hospital de día de adolescentes.

\section{Hospital de Día para adolescentes}

Atiende pacientes de 13 a 18 años que han sido derivados desde los equipos de atención a niños y adolescentes de los centro de salud mental. La población en edad escolar recibe apoyo de un equipo de educación, durante una tercera parte de la jornada. La plantilla sanitaria integra una psiquiatra, un psicólogo, un enfermero, un terapeuta ocupacional y un monitor de terapia ocupacional. El equipo docente está conformado por una coordinadora, dos profesores y una profesora técnica de servicios a la comunidad, que dependen de la Consejería de Educación. El apoyo administrativo y logístico se comparte con el equipo de adultos.

\section{Dispositivos de rehabilitación}

Hasta el año 1987 en la Consejería de Salud y Social existieron dos direcciones generales correspondientes a cada una de estas áreas. El "Programa de Recursos Alternativos a la Hospitalización Psiquiátrica” era común a las dos. Cuando las competencias se separaron en dos consejerías el programa pasó a depender de la de Integración Social. En el año 1988 se creó el primer dispositivo de rehabilitación psicosocial dependiente de la entonces Consejería de Integración Social. Los dispositivos de rehabilitación han sido desarrollados en la mayor parte de Madrid desde la Consejería que se ha hecho cargo de Servicios Sociales y no desde la de Sanidad y lo han hecho como un servicio público, integrado en la red de atención a la salud mental, pero en el que actúan como proveedoras entidades privadas que contratan equipos multiprofesionales que integran psicólogos y profesionales de apoyo (Bravo Ortiz, Díaz Mújica, \& Fernández Liria, 2006).

\section{Centros de Rehabilitación Psicosocial}

El objetivo de los Centros de Rehabilitación Psicosocial (CRPS) es ayudar a las personas con enfermedad mental grave y crónica a recuperar el máximo grado de autonomía personal y social, mejorar su funcionamiento psicosocial y promover su integración en la comunidad así como apoyar a sus familias. Como el resto de los dispositivos de rehabilitación y apoyo, atienden pacientes que han sido derivados desde los Centros de Salud Mental y que mantienen en éste su psiquiatra y su coordinador de cuidados de referencia. Ofrecen programas individualizados de apoyo a la rehabilitación psicosocial y promoción de la integración social, desarrollando intervenciones individuales y grupales en las siguientes áreas: autocuidado y actividades de la vida diaria, psicomotricidad, habilidades sociales, autocontrol, psicoeducación y manejo de la enfermedad, integración comunitaria y apoyo a la integración social. Así mismo ofrecen psicoeducación, asesoramiento y apoyo a las familias. Proporcionan planes individualizados de rehabilitación psicosocial y apoyo a la integración social a pacientes graves que, por requerirlos, han sido derivados desde los centros de salud mental. Funcionan de 09:00h. a 18:00h. como un programa de día.

Existen dos CRPS con 90 plazas uno y 60 otro. La plantilla de cada uno de estos dos centros está integrada por un psicólogo director, dos o tres psicólogos de plantillas, dos o tres educadores, un terapeuta ocupacional, un trabajador social y el apoyo administrativo y logístico. Son centros propios de la Dirección General de Servicios Sociales de la Consejería de Familia y Asuntos Sociales, en el marco de la Red Pública de Atención Social a personas con enfermedad mental grave y duradera.

\section{Centros de Rehabilitación Laboral}

El objetivo de los CRL es ayudar a aquellas personas con enfermedad mental grave que han alcanzado un buen nivel de autonomía, a aprender o recuperar los hábitos y capacidades laborales necesarias para acceder al mundo laboral y apoyar su integración y mantenimiento en el mismo. Se ofrecen un abanico variado de actividades y programas tanto individuales como grupales: orientación vocacional, entrenamiento en hábitos básicos de trabajo, ajuste socio- laboral, talleres pre-laborales, apoyo a la formación laboral, búsqueda activa de empleo, apoyo y seguimiento de la inserción laboral. Funcionan de 09:00h. a 18:00h.

Se dispone de 2 CRL en el área con una capacidad de 50 y 30 plazas y una plantilla tipo de Director (psicólogo), 1 psicólogo, 1 Terapeuta Ocupacional, 1 Técnico de Apoyo a la Integración Laboral, 1 Preparador Laboral, 3 Maestros de Taller y el apoyo administrativo y logístico. Son centros propios de la Dirección General de Servicios Sociales de la Consejería de Familia y Asuntos Sociales, en el marco de la Red Pública de Atención Social a personas con enfermedad mental grave y duradera.

\section{Centros de Día}

Ofrecen programas y actividades de apoyo y soporte social, para ayudar a las personas con enfermedad mental grave y con mayores niveles de dificultades de funcionamiento, aislamiento y dependencia, a alcanzar un mínimo de autonomía y apoyar su mantenimiento en la comunidad en las mejores condiciones posibles. Ofrecen para ello actividades de mantenimiento de la autonomía personal, actividades de socialización, actividades de ocio y actividades socioculturales de soporte social como las siguientes: manualidades, pintura, juegos de mesa, tertulias, elaboración de periódico, taller de lectura y escritura, taller de creatividad, expresión corporal, gimnasia, y cuantas otras actividades sociales, ocupacionales o culturales puedan resultar relevantes. La meta del Centro de Día es la estructuración de la vida de la persona y ayudar a su mantenimiento en las mejores condiciones y buscar en la medida de lo posible la mejora de su autonomía y posibilidades de integración social. Existen tres Centros de Día en la zona con 30 plazas cada uno. La plantilla de cada uno de estos tres centros está integrada por un psicólogo director, un psicólogo de plantilla, dos educadores, terapeuta ocupacional y el apoyo administrativo y logístico. Funcionan de 09:00h. a 18:00h. como un programa de día. Dos de estos centros son propios de la Dirección General de Servicios Sociales de la Consejería de Familia y Asuntos Sociales, en el marco de la Red Pública de Atención Social a personas con enfermedad mental grave y duradera. El otro es concertado y financiado por la Consejería de Familia y Servicios Sociales.

Equipos de apoyo social y comunitario 
Los equipos de apoyo social y comunitario (EASC) se dirigen a personas con enfermedades mentales graves con dificultades de integración en recursos socio-comunitarios e importantes necesidades de soporte social y familiar; que necesitan una atención flexible y cercana en su propio domicilio a fin de prevenir situaciones de abandono y marginación y sobre todo ayudarles en la medida de lo posible a mantenerse en adecuadas condiciones en su propia vivienda y/o en su entorno familiar y social. Ofrecen atención social domiciliaria y en el propio entorno a aquellas personas con enfermedad mental grave y con dificultades y necesidad de apoyo social para poder mantenerse en su entorno familiar y comunitario. Existen dos EASC en la zona con 30 plazas cada uno. La plantilla de cada equipo está formada por un trabajador social, un psicólogo y dos educadores. Funcionan de 09:00h. a 18:00h. Están concertados y financiados por la Consejería de Familia y Servicios Sociales, en el marco de la "Red pública de atención social a personas con enfermedad mental".

\section{Minirresidencias}

Las miniresidencias constituyen un servicio residencial flexible y polivalente de entre 20 y 30 plazas. Ofrece estancias cortas, transitorias o indefinidas para atender diferentes necesidades. Ofrecen: alojamiento y manutención; atención psicosocial y apoyo personal para la mejora de la autonomía y para apoyar su integración y supervisión las 24 horas del día. La población diana a atender en las miniresidencias son las personas con enfermedades mentales graves que necesitan un apoyo residencial específico de mayor o menor duración, y con supervisión de 24 horas debido a la ausencia o sobrecarga de la familia para su atención y cuidado o por presentar problemas de autonomía y funcionamiento psicosocial que les impidan o dificulten para cubrir autónomamente sus necesidades de alojamiento y soporte.

Existen 2 miniresidencias con 30 y 25 plazas en la zona. La plantilla de cada residencia está compuesta por un psicólogodirector, 1 psicólogo de plantilla, 1 terapeuta ocupacional, 13/15 educadores, y el apoyo administrativo y logístico. Una de las miniresidencias es un centro propio de la Dirección General de Servicios Sociales de la Consejería de Familia y Asuntos Sociales, en el marco de la "Red Pública de Atención Social a personas con enfermedad mental grave y duradera". La otra es concertada y financiada por la Consejería de Familia y Servicios Sociales.

\section{Piso escuela}

Alternativa de alojamiento estable y normalizada organizada en un piso comunitario en la que residen 3 ó 4 personas con trastorno mental grave y que es utilizado para estos $\mathrm{u}$ otros usuarios como contexto de aprendizaje de las habilidades de manejo en el entorno residencial. De esta forma se organizan actividades y programas de compra, cocina, cuidado de la habitación, etc. supervisadas por un profesional. Se dispone de 1 piso escuela en la zona y su dependencia es de la Consejería de Sanidad.

\section{Pisos protegidos}

Alternativa de alojamiento estable y normalizada organizada en pisos normales en los que residen 3 ó 4 personas con enfermedad mental recibiendo el apoyo y supervisión que en cada momento necesiten. Los pisos supervisados funcionan vinculados y en coordinación con las residencias. Los pisos supervisados están diseñados para personas con enfermedades mentales graves y crónicas que tienen un nivel mínimo de autonomía personal y social pero necesitan un apoyo residencial debido a: ausencia de apoyo familiar o problemas de autonomía que les impidan o dificulten para cubrir autónomamente sus necesidades de alojamiento y soporte. No requieren una supervisión de 24 horas como la que ofrecen las Residencias. Los pisos supervisados funcionan vinculados a las miniresidencias. En la actualidad se dispone de 3 pisos con 12 plazas totales. Al menos hay un educador de apoyo para la supervisión de cada piso. Estos educadores para pisos se añaden y son complementarios a las plantillas de la minirresidencia.

\section{Dispositivos de Apoyo}

\section{Clubes sociales}

Se trata de clubes que ofertan actividades de ocio a pacientes que, por sus características, no pueden realizar estas en un entorno más normalizado. Se accede a ellos por indicación de los centros de salud mental. Se mantienen en base a personal voluntario con un apoyo profesional desde el centro de salud mental.

\section{Huerto de Ocio}

Proporciona una actividad ocupacional o prelaboral, instrumentada a través de diferentes recursos de rehabilitación y centros de salud mental del área. Se desarrolla en un terreno cedido a la asociación de familiares de pacientes mentales (APISEP) por la Consejería de Medio Ambiente de la Comunidad de Madrid.

\section{Empleo protegido}

En diferentes momentos ha habido distintas alternativas de empleo para personas con trastorno mental grave apoyadas desde el sistema de salud mental. En la actualidad en uno de los municipios menores del área se sitúa una empresa social de manipulado farmacéutico secundario. En el momento actual la empresa da trabajo a 76 pacientes.

\section{Programa de Continuidad de Cuidados}

El programa de continuidad de cuidados tiene como objetivo facilitar la actuación coordinada y sinérgica de las distintas unidades y programas de los servicios de salud mental y de otros recursos comunitarios sobre aquellas personas que tienen dificultad para extraer beneficio de los mismos bien porque requieren simultánea o sucesivamente el uso de varios de ellos, o bien porque carecen de los aportes sociales necesarios. Para ello procura proporcionar a estas personas un sistema adecuado de cuidados mediante la asignación de un coordinador de cuidados y elaboración y desarrollo de un plan individualizado que garantice la atención de las necesidades detectadas y el funcionamiento sinérgico de todos los recursos que se destinen a satisfacerlas. Los profesionales que actúan como coordinadores de cuidados en 
este programa son 6 diplomados en enfermería y 4 trabajadores sociales que tienen su base en los centros de salud mental y actúan en estrecha colaboración con los equipos de apoyo social y comunitario. Los usuarios atendidos en el programa son, sobre todo, pacientes con trastorno mental grave.

El programa de coordinación de cuidados es la forma que empezaron adoptando en Madrid (desde 1989 en Leganés y desde 1991 en éste área) los programas por los que se presta especial atención a los pacientes graves y crónicos (Fernández Liria \& García Rojo, 1990). La Estrategia en Salud Mental del Sistema Nacional de Salud español (Ministerio de Sanidad, 2007) recomienda la puesta en marcha de estos programas y cita el de esta área como ejemplo de buena práctica y ejemplo de uno de los tres modelos que se están desarrollando en el país (Los otros son el de tratamiento asertivo comunitario y uno mixto (Alonso Suárez et al., 2004). Estos programas han demostrado ser eficaces al menos en la reducción de ingresos y estancias hospitalarios y en la reducción del número de urgencias (Alonso Suárez et al., 2011).

\section{Financiadores y proveedores}

La articulación de la red de servicios descrita en el apartado anterior se guió por un proyecto de servicios de salud mental que modelo intenta emular la red de servicios comunitarios que resultó de la transformación de uno de los dos hospitales psiquiátricos públicos que hasta la década de los 1980 existían en Madrid (Desviat, 1992, 1994, 2001, 2007).

La principal diferencia entre lo entonces ya logrado en Leganés y lo entonces sólo proyectado en Alcalá de Henares era que el proyecto de Leganés se basó en la reutilización de recursos públicos anteriormente adscritos al hospital psiquiátrico.
El resultado fue una red de servicios públicos, construida con recursos de titularidad pública y con una capacidad de gestión heredada de la dirección y administración del antiguo hospital.

En la zona Este de Madrid, el plan parte de la práctica inexistencia de recursos públicos, con lo que, cuando veinte años después, se ha articulado una red que funcionalmente es muy parecida a la de Leganés, se ha tenido que hacer a base de integrar recursos que tienen financiadores distintos (aunque siempre públicos), dispensan servicios gestionados por proveedores diferentes (a veces privados) y tienen dependencias administrativas también diferentes con lo que la articulación de un procedimiento de dirección y gestión ha de resultar de un acuerdo entre socios que ha de estar en continua revisión. La figura 3 representa a los principales financiadores de la red que son básicamente la Consejería de Sanidad, La Consejería de Familia y Servicios Sociales, el Ayuntamiento de Alcalá de Henares y el Plan Regional de Drogodependencias. Hay recursos con financiaciones, ad hoc, y financiadores variables, como los clubs sociales, el huerto de ocio o el empleo protegido.

Como puede observarse no sólo la red en sí, sino algunos de sus nudos (por ejemplo, el piso escuela) existen como consecuencia de la acción coordinada de recursos con financiadores diferentes. La figura 4 representa a los proveedores que pueden ser varios para cada uno de los financiadores.

\section{Estructura funcional}

La única posibilidad de logar el funcionamiento coordinado de recursos con dependencia tan variada es partir de un acuerdo entre los financiadores para garantizarla. La figura 5 representa el organigrama de la red. Como puede verse en ella, la cúspide de la organización está constituida por un Consejo de Financiadores

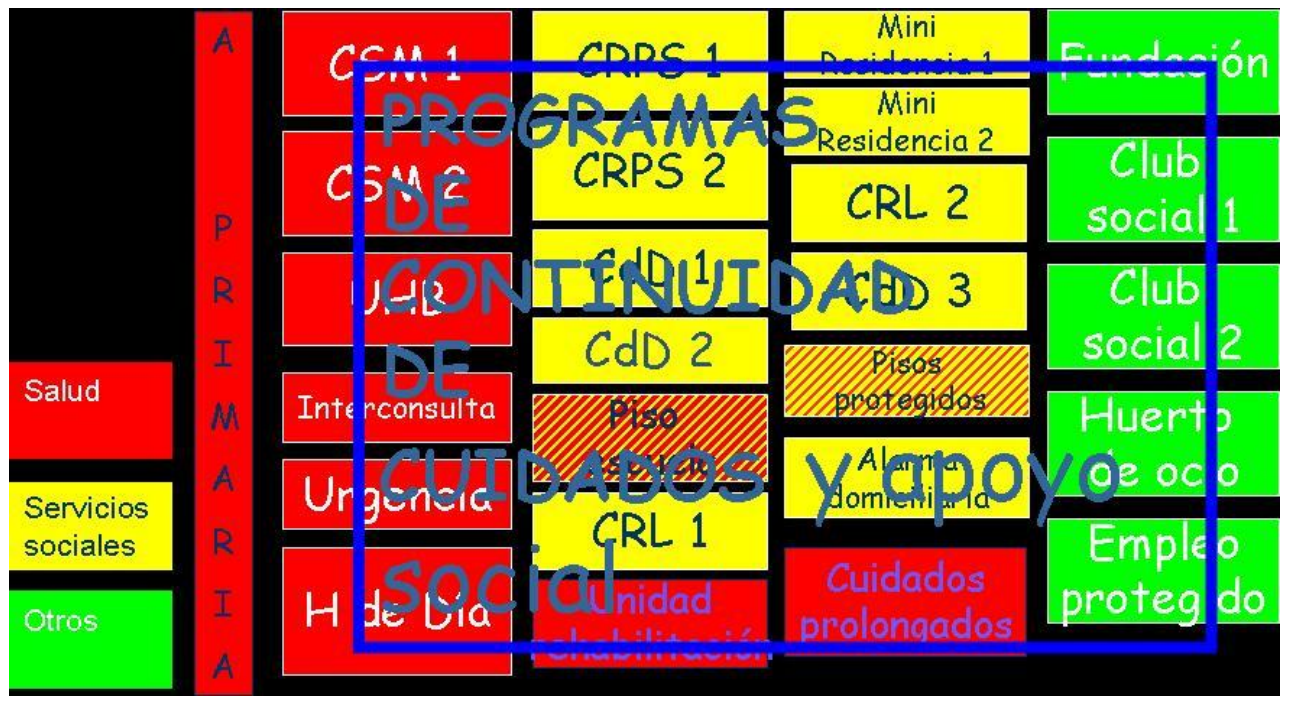

Figura 3. Principales financiadores de la red.

(antes se llamó Comité de Enlace) en el que los representantes de las instituciones financiadores marcan los objetivos generales para delegar su puesta en marcha en la estructura funcional.

El Consejo de Financiadores delega sus atribuciones y hace depositario de sus directrices a un responsable de los servicios que actúa como presidente de la Comisión de Asistencia que integra a los responsables de todos los dispositivos de la red y es el máximo órgano de dirección profesional.

La Comisión de Asistencia se diferencia de las otras comisiones técnicas en que está constituida por los responsables de los dispositivos (en principio nombrados por las entidades proveedoras) mientras que el resto de las comisiones técnicas 


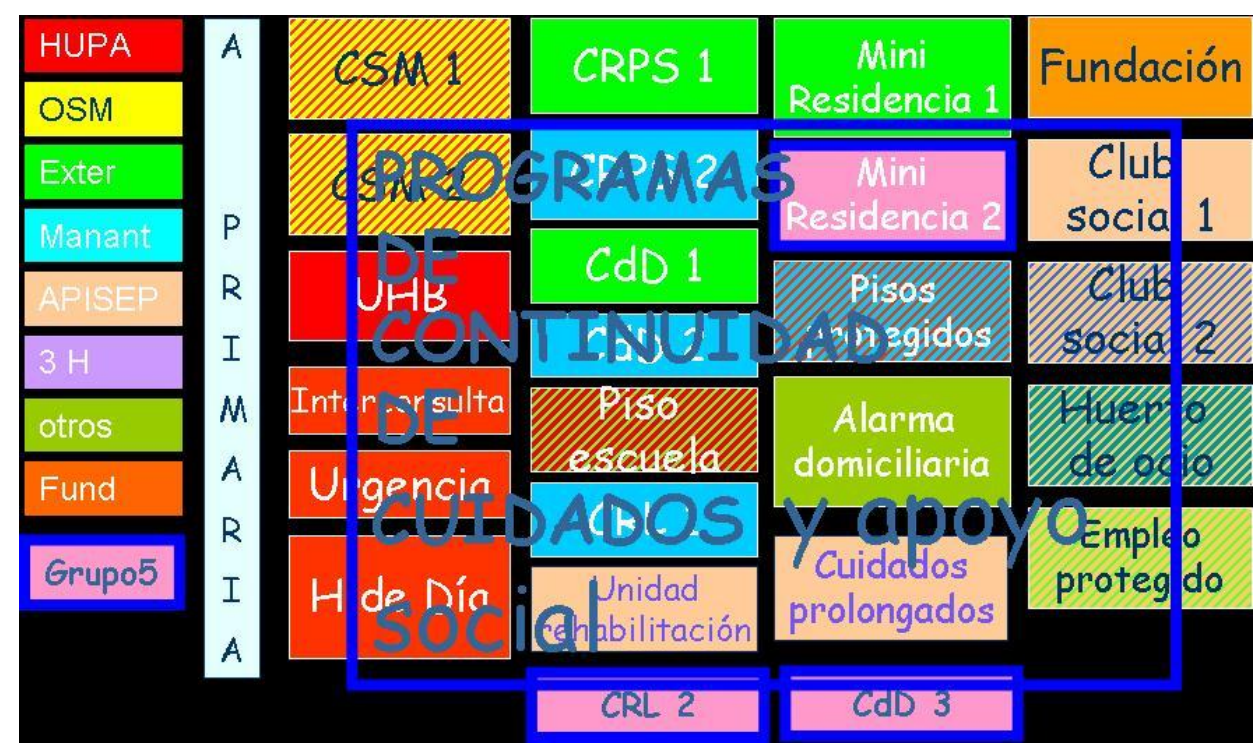

Figura 4. Principales proveedores de la red

(pensadas para atender funciones cuya complejidad requiere de un grupo de trabajo) están integradas por representantes de los dispositivos elegidos por los equipos interprofesionales de éstos.

De entre las comisiones merece mención especial la de formación que, en realidad integra tres funciones: 1) la de procurar la necesaria formación continuada al personal de plantilla, 2) la de atender los compromisos de los servicios con la enseñanza universitaria de pre y postgrado en la Facultad de Medicina y la Escuela de Enfermería de la Universidad de Alcalá y 3 ) la de organizar la formación de especialistas en Psiquiatría, Psicología Clínica y Enfermería Especialista en salud mental. La red está acreditada para la formación de 16 residentes de Psiquiatría (4 por cada uno de los cuatro años que dura la residencia), 16 residentes de Psicología Clínica (4 por año) y 12 de Enfermería Especialista en Salud mental (6 por cada uno de los dos años que durará la residencia).

\section{Balance y perspectivas}

Los Servicios de Salud mental correspondientes al Hospital Universitario Príncipe de Asturias constituyen una red de servicios que integra numerosos dispositivos desde los que prestan sus servicios diversos equipos interprofesionales guiados por el propósito común de satisfacer las necesidades de atención en materia de salud mental de una población de 450.000 habitantes de un área geográfica delimitada. Como corresponde a las prestaciones del Sistema Nacional de Salud español, su atención es universal (Incluye a toda la población del área de referencia) y gratuita en el momento del uso. El sistema de atención especializada funciona en segunda línea respecto a la atención primaria de salud y, generalmente, por indicación de ésta. Los servicios cumplen, además, una importante labor docente y realizan trabajo de investigación asistencial y básica. Esto es posible aunque los recursos dependen de organismos diferentes y tienen vinculaciones diferentes con el sector público, gracias a la existencia de unos acuerdos que permiten mantener una estructura funcional conjunta.

En la actualidad en la Comunidad de Madrid está en marcha un proceso de transformación del sistema sanitario promovido por el gobierno conservador que se orienta a facilitar la introducción de mecanismos de financiación y gestión privados en un sistema tradicionalmente público y en el establecimiento de medidas que pretenden flexibilizar la adscripción territorial de las responsabilidades de atención sanitaria y promover la competencia entre dispositivos. Aunque la propia existencia de la red (en la que coexisten proveedores privados con los públicos) demuestra que alguna de estas medidas no tienen por qué conllevar una amenaza para el sistema, está por comprobar el modo en el algunas otras (como la dilución de las responsabilidades territoriales) puede afectar a la atención integral a la salud mental de la población.

\section{Referencias}

Alonso Suárez, M., Bravo Ortiz, M. F., \& Fernández Liria, A. (2004). Origen $\mathrm{y}$ desarrollo de los programas de seguimiento y cuidados para pacientes mentales graves y crónicos en la comunidad. Revista de la Asociación Española de Neuropsiquiatría, 24, 25-51.

Alonso Suárez, M., Bravo-Ortiz, M., Fernández-Liria, A., \& González-Juárez, C. (2011). Effectiveness of Continuity-of-Care Programs to reduce time in hospital in persons with schizophrenia. Epidemiology and Psychiatric Sciences, 20, 1-8.

Bravo Ortiz, M., Díaz Mújica, B., \& Fernández Liria, A. (2006). La atención a los trastornos mentales graves. In F. Pérez (Org.), Dos décadas tras la reforma psiquiátrica (pp. 277-296). Madrid: AEN.

Desviat, M. (1992). La reforma del hospital psiquiátrico: del hospital al área de salud. Psiquiatría Pública, 4, 3-16.

Desviat, M. (1994). La reforma psiquiátrica. Madrid: DOR.

Desviat M. (2000) ¿Hacia dónde la reforma? I Congreso Virtual de Psiquiatría. Recuperado de http://www.dinarte.es/salud-mental/experiencia/48_c.htm

Desviat, M. (2001). Salud pública y psiquiatría. Revista de la Asociación Española de Neuropsiquiatría, 21(77), 125-133.

Desviat, M. (2007). De locos a enfermos: de la psiquiatría del manicomio a la salud mental comunitaria. Madrid: Ayuntamiento de Leganés.

Fernández Liria, A., \& García Rojo, M. J. (1990). Los programas de "Case Management". Conceptos basicos y aplicabilidad. Revista de la Asociación Española de Neuropsiquiatría, 10(32), 65-75. 
Goldberg, D., \& Huxley, P. (1980). Mental illness in the community: the pathway to psychiatric care. London: Tavistock.

Goldberg, D., \& Huxley, P. (1992). Common mental disorders: a bio-social model. London: Routledge.

Hornillos Jerez, C., Rodríguez Pedraza, E., González Aguado, F., \& Fernández Liria, A. (2009). Presencia física de profesionales de salud mental en un centro de atención primaria como forma alternativa de coordinación: una experiencia piloto. Revista de la Asociación Española de Neuropsiquiatría, 29(104), 355-370.

Kessler, R., \& Üstün, T. (2008). The WHO World Mental Health Survey: global perspectives on the epidemiology of mental disorders. New York: Cambridge University Press.

Ley n. 14 de 25 de abril de 1986. Ley General de Sanidad. Boletín Oficial del
Estado, 29/04/1986

Ministerio de Sanidad y Consumo (2007). Estrategia en Salud Mental del Sistema Nacional de Salud. Madrid: Autor.

Ministerio de Sanidad y Política Social (2009). Contenido de la Cartera de Servicios comunes del Sistema Nacional de Salud y Procedimientos para su actualización. Recuperado de http://www.msps.es/profesionales/ prestacionesSanitarias/publicaciones/docs/carteraServicios.pdf

Salvador-Carulla, L., Bulbena, A., Vázquez-Barquero, J. L., Muñoz, P. E., GómezBeneyto, M., \& Torres, F. (2002). La Salud Mental en España: ¿Cenicienta en el País de las Maravillas? In: J. M. Cabasés, J. R. Villalba, \& C. Aibar (Orgs.), Informe SESPAS 2002: Invertir para la Salud. Prioridades en Salud Pública (pp. 301-326). Valencia: Generalitat Valenciana y Escuela Valenciana de Estudios para la Salud.

Alberto Fernández Liria é psiquiatra no Hospital Universitario Príncipe de Asturias/Universidad de Alcalá de Henares. Endereço para correspondência: C/ Fernán González 79, 6․ 28009, Madrid. Telefone: +34 91 88781 00, extensión 2523. E-mail: afliria@gmail.com

Ana Moreno Pérez é psiquiatra no Hospital Universitario Príncipe de Asturias. E-mail: anamorenoperez@ gmail.com

Juan González Cases é psicólogo do Centro de Rehabilitación Psicosocial de Alcalá de Henares. Consejería de Familia y Asuntos Sociales. Comunidad de Madrid (Gestión técnica Grupo Exter S.A.).E-mail: jgcases@ wanadoo.es 